\title{
Supernova remnants in clumpy media: propagation of accelerated particles into clumps
}

\author{
Silvia Celli* \\ Gran Sasso Science Institute, Viale Francesco Crispi 7, 67100 L'Aquila, Italy \\ E-mail: silvia.celliegssi.infn.it

\section{Felix Aharonian} \\ Gran Sasso Science Institute, Viale Francesco Crispi 7, 67100 L'Aquila, Italy \\ Dublin Institute for Advanced Studies, 31 Fitzwilliam Place, Dublin 2, Ireland \\ Max-Planck-Institut für Kernphysik, Postfach 103980, D-69029 Heidelberg, Germany \\ E-mail: felix.aharonian@mpi-hd.mpg.de
}

\section{Stefano Gabici}

APC, AstroParticule et Cosmologie, Universitè Paris Diderot, CNRS/IN2P3, CEA/Irfu, Obs de Paris, Sorbonne Paris Citè, France

E-mail: gabicieapc.in2p3.fr

\section{Giovanni Morlino}

Gran Sasso Science Institute, Viale Francesco Crispi 7, 67100 L'Aquila, Italy

E-mail: giovanni.morlinodgsi.infn.it

\begin{abstract}
Supernova remnant (SNR) shocks are sites of particle acceleration, as indicated by observations from the radio to the gamma-ray domain. In particular, gamma-rays can be produced as the result of the interaction between particles accelerated at the SNR shock and the ambient matter and/or radiation. Both protons and electrons can contribute to the observed gamma-ray emission from SNRs, through neutral pion decay and inverse Compton (IC) scattering, respectively. Ascribing the origin of the SNR gamma-ray emission to either hadronic or leptonic processes remains, in most cases, an open problem, and its solution would constitute a crucial step in the quest for cosmic-ray (CR) sources. It has been proposed that the presence of dense clumps in the environment where supernovae explode can have a dramatic impact in shaping the hadronic gamma-ray spectrum. This is because the high-energy protons accelerated at the SNR shock are able to penetrate the dense clumps while low-energy ones cannot, and thus probe the diffuse inter-clump medium only. Here we present a numerical study of the penetration of relativistic protons into clumps which are engulfed by a SNR shock. We show that the spectrum of protons inside clumps is much harder than that in the diffuse inter-clump medium and we discuss the implications for the production of hadronic gamma-rays.
\end{abstract}

35th International Cosmic Ray Conference - ICRC2017

10-20 July, 2017

Bexco, Busan, Korea

${ }^{*}$ Speaker. 


\section{Introduction}

It is now well established that supernova remnants (SNR) can accelerate both leptons and hadrons, as demonstrated by the non thermal emission detected from several SNRs. In particular the pion-bump detected at $\approx 280 \mathrm{MeV}$ from two middle aged SNRs, IC443 and W44, indicates the presence of accelerated hadrons [1]. Nevertheless two main questions need to be answered in order to validate the idea that SNRs are the main source of the galactic CRs observed at Earth: which is the total amount of energy channeled into relativistic particles and which is the final spectrum injected into the interstellar medium (ISM). Gamma-ray observations provide a privileged tool to answer these questions, allowing to directly infer the properties of accelerated hadrons, something that cannot be done in any other wavelength.

In some young SNRs it is still unclear whether the detected gamma-ray emission is produced by leptonic process - via IC scattering - or hadronic collisions - through the decay of resulting neutral pions. On a general ground, the detection of hadronic emission would favor the high efficient acceleration scenario, while the leptonic interpretation forces one to assume a quite low acceleration efficiency. This difference is mainly due to the different magnetic field strength required: IC scenario usually needs very low magnetic field (of the order of $10 \mu \mathrm{G}$ ) in order to simultaneously account for radio, $\mathrm{X}$-ray and $\gamma$-ray emission. On the contrary, hadronic scenarios requires much larger values, of the order of few hundreds of $\mu \mathrm{G}$, that cannot result from the simple compression of interstellar magnetic field, but requires some sort of amplification, which is, in turn, a possible signature of efficient CR acceleration itself. The case of RX J1713.7-3946 is of special interest to this respect. This remnant has been considered for long time the best candidate for an efficient acceleration scenario, mainly due to its high $\gamma$-ray luminosity. The detection of $\gamma$-ray emission in the energy range [1-300] GeV by the Fermi-LAT satellite [2] has shown an unexpected hard spectrum which, at a first glance, seems to be more in agreement with a leptonic scenario. Nevertheless, a deeper analysis shows that neither the hadronic nor the leptonic scenarios, taken in their simplest form, can unequivocally explain the observations [3-5], hence the issue remains open. In particular is has been proposed $[3,6,7]$ that an harder spectrum could result also from hadronic emission if the SNR is expanding inside a clumpy medium. In such a case, in fact, hydrodynamical instabilities resulting from the shock-clump interaction can amplify the magnetic field all around the clump, making difficult for particles at low energies to penetrate inside the clump compared to the most energetic ones. The final result would be a $\gamma$-ray spectrum harder than the parent proton spectrum.

In this work we investigate exactly such a scenario, solving the 3D time-dependent transport equation for CRs in the shock vicinity, when the shock crosses a dense clump, as introduced in Sec. 2 and discussed in Sec. 3. The diffusion coefficient around the clump is parametrized in order to mimic the amplified magnetic field resulting from magneto-hydrodynamic (MHD) instabilities. The spatial and temporal behavior of the CR density is obtained in Sec. 4, and the resulting CR spectrum from inside and outside the clump region is calculated, showing that the spectrum inside the clump is much harder at early times and steepens at later times. We finally discuss the detectability of clumps-shock association, through the related gamma-ray emission from protonproton interaction in Sec. 5. 


\section{Modelling the transport of cosmic-rays at astrophysical shocks propagating in clumpy media}

The transport equation describes the time and space evolution of the density of cosmic-rays in the phase space $f(\vec{x}, \vec{p})$, as a result of the action of different processes, namely advection into a shock, diffusion in turbulent magnetic field, adiabatic compression due to the presence of a shock, energy losses and CR sources. Such equation reads as:

$$
\frac{\partial f}{\partial t}+\bar{u} \cdot \bar{\nabla} f=\bar{\nabla} \cdot[D \bar{\nabla} f]+\frac{1}{3} p \frac{\partial f}{\partial p} \bar{\nabla} \cdot \bar{u}-\frac{1}{p^{2}} \frac{\partial}{\partial p}\left[\dot{p} p^{2} f\right]+Q \delta(\bar{x})
$$

where $D=D(\vec{x})$ is the diffusion coefficient, $\vec{u}$ is the background plasma speed, $\dot{p}$ is the momentum loss and $Q$ is the source term. The shock is assumed to be moving along the $z$-coordinate: the space region in front of the shock is called upstream region, while the region that has been advected by the shock is called the downstream. We assume a Bohm diffusion coefficient, $D(\vec{x}) \propto E / B(\vec{x})$, as appropriate in highly turbulent environments such as shocks.

\subsection{Molecular clump}

A molecular clump is a dense and cold $H_{2}$ region of the ISM, with a typical density of $n_{c} \in$ $\left[10^{3}-10^{5}\right] \mathrm{p} / \mathrm{cm}^{3}$ and a temperature of $T_{c} \simeq 10 \mathrm{~K}$. In the following, the clump is considered to be spherical with a radius $R_{c}=0.1 \mathrm{pc}$. The clump is surrounded by a region of size $\approx R_{c} / 2$ where the magnetic field is highly amplified due to the shock-clump interaction (see e.g. [6]). We assume a magnetic field of strength $10 \mu \mathrm{G}$ throughout the simulation box, except for the region surrounding the clump, where it is set to $100 \mu \mathrm{G}$ to mimic the field amplification. On the other hand, inside the clump the diffusion coefficient is expected to be significantly larger, due to severe damping of MHD turbulence induced by ion-neutral friction (e.g. [8]). For this reason, a magnetic field $B_{c}=1 \mu \mathrm{G}$ is assumed inside the clump.

\subsection{The velocity field}

The clump is considered as a solid body, that the plasma is not able to penetrate. The plasma, on the other hand, is assumed to be an incompressible fluid. Under these hypotheses, the spatial dependence of the downstream velocity field around a clump is obtained analytically as a solution of the Laplace equation, with the following boundary conditions:

1. the velocity field is uniform at large distances from the clump: it assumes the value $\vec{u}=$ $u_{0} \hat{z}$, where $u_{0}$ is the plasma speed in the downstream region, set to $u_{0}=3.3 \times 10^{8} \mathrm{~cm} / \mathrm{s}$, as expected in RX J1713.7-3946 [7];

2. a no-slip condition $(\vec{u} \cdot \hat{n}=0)$ is imposed at the boundary among the clump and the ISM, which implies that the filed is directed along the tangential direction. 
The field is also assumed independent on the azimuthal angle. The solution obtained in cylindrical coordinates reads as

$$
\begin{aligned}
& u_{r}(r, z)=-\frac{3}{2} u_{0} \frac{R_{c}^{3} r z}{\left(r^{2}+z^{2}\right)^{5 / 2}} \\
& u_{z}(r, z)=u_{0}+\frac{1}{2} u_{0} R_{c}^{3} \frac{r^{2}-2 z^{2}}{\left(r^{2}+z^{2}\right)^{5 / 2}}
\end{aligned}
$$

where the $r$ axis originates at the centre of the clump. This solution has null divergence outside the clump. Moreover, the velocity field is set to zero inside the clump. Indeed the shock penetrates the clump at a much lower speed with respect to its speed in the ISM, as shown in [9]: if the clump density is $\rho_{c}$ and the ISM density is $\rho_{I S M}$, then the shock speed in the clump $u_{c}$ is related to the shock speed in the ISM $u_{s}$ by the energy-momentum conservation law, therefore

$$
u_{c}=\sqrt{\frac{\rho_{I S M}}{\rho_{c}}} u_{s} \longrightarrow u_{c}<<u_{s}
$$

which justifies our assumption of a virtually unshocked clump. Finally, the velocity field is set to zero also in the upstream region.

\section{Numerical integration of the transport equation}

The transport equation is solved in the upstream reference frame, where the shock position evolves with time as $z_{s}=u_{s} d t$, while the clump is assumed to be at rest in the centre of the box. We consider only CR protons and therefore we can neglect energy losses, mainly due to protonproton interaction, since they operate on a time scale which is longer than the remnant age. We also neglect the adiabatic compression term, since we use a divergence-less velocity field, as described in Sec. 2.2. For convenience, Eq. 2.1 can be rewritten in cylindrical coordinates as

$$
\frac{\partial f}{\partial t}=\frac{1}{r} \frac{\partial}{\partial r}\left[r D(r, z) \frac{\partial f}{\partial r}\right]+\frac{\partial}{\partial z}\left[D(r, z) \frac{\partial f}{\partial z}\right]-u_{r}(r, z) \frac{\partial f}{\partial r}-u_{z}(r, z) \frac{\partial f}{\partial z}+Q(p) \delta\left(z-z_{s}\right)
$$

The source term simulates $\mathrm{CR}$ injection at the shock position $z=z_{s}$, with an unbroken power law spectrum in momentum $f(p) \propto p^{-4}$, as resulting from Diffusive Shock Acceleration [10,11] mechanism. This equation is solved through an implicit method, that relies on the operator splitting technique in order to solve simultaneously for the $r$ and $z$ dimensions. The integration method used is the so-called Alternating Direction Implicit (ADI): the diffusive part of the equation is discretized in a flux conservative way, while the advective part is written in an upwind setting. The method is second order in both time and space.

\section{Results}

The transport equation is solved separately for CR protons of energy ranging from $E_{p}=1 \mathrm{GeV}$ up to $E_{p}=1 \mathrm{PeV}$. The most remarkable feature of its solution is that low-energy CRs are prevented from penetrating the clump because of the high magnetic field that they encounter in the clump layer as well as because of the strong energy dependence of the Bohm diffusion coefficient, which 
makes the diffusion time for penetrating the clump layer much longer for lower energies. The effect is well visible in Fig. 1(a) to Fig. 1(f), where the shock profiles at a fixed $r$ inside the clump are shown for different $\mathrm{CR}$ proton energies at different times. The amount of particles inside the clump is a function of time: in this regard, an important time scale of the system is the time $t_{c}$ at which the shock starts penetrating the clump. While high-energy CRs soon start to fill the clump, the low-energy ones are able to enter the clump only at much later times. A feature in the shock profile is visible for primary energies dominated by diffusion, for instance when $E_{p}>10 \mathrm{TeV}$ : the enhancement observed in Fig. 1(e) and 1(f) at times immediately after $t_{c}$ is due to the fact that particles are prevented to penetrate the clump due to the high magnetic field present in the clump layer. Low-energy primaries however do not experience it since their evolution is dominated by advection, so that they can freely move around the clump.

\subsection{Proton spectrum inside and outside the clump}

Given the solution of Eq. 3.1, it is straightforward to derive the proton spectrum $J(p)$ at different times, from both inside and outside the clump. The spectrum of protons inside the clump, defined as the number of particles per unit surface, time, solid angle and three dimensional momentum, reads as

$$
J_{c}(p)=\frac{c}{4 \pi V_{c}} \frac{d^{3} N_{c}}{d p^{3}}
$$

where $V_{c}=\frac{4}{3} \pi R_{c}^{3}$ is the clump volume and $d^{3} N_{c}$ is the number of CRs with momentum between $p$ and $p+d p$ penetrating the clump at a given time $t$, which is obtained as

$$
d^{3} N_{c}=2 \pi \int_{r^{2}+z^{2} \leq R_{c}^{2}} r d r d z \int 4 \pi p^{2} f(r, z, p) d p
$$

The same procedure can be applied to compute the spectrum emitted from the region outside the clump, accounting for the remaining volume of the grid.

At any fixed time within about 200 yrs from the time at which the shock enters the clump, the spectrum from inside the clump shows an evident hardening with respect to the spectrum from the outside region, as reported in Fig. 2(a) and Fig. 2(b). It is well visible that the slope of the spectrum from inside the clump strongly depends on the time of observation.

This behavior will impact on the gamma-ray emission of the remnant, which is dominated by its densest regions, therefore by the clumpy structures: clumps that have just been shocked will produce gamma-rays with a harder spectrum than the ones deriving from older clumps. It is therefore crucial to consider the contribution from a given spatial distribution of clumps in order to conclusively evaluate the effect of the presence of molecular clumps in a shock environment on the resulting hadronic gamma-ray emission. This will be the object of a future work. 


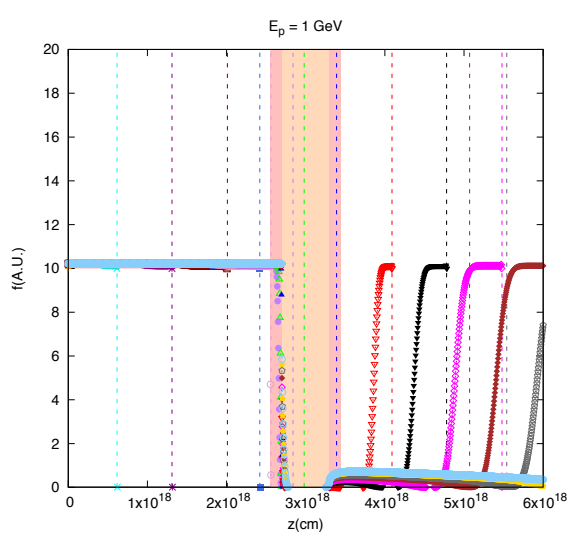

(a)

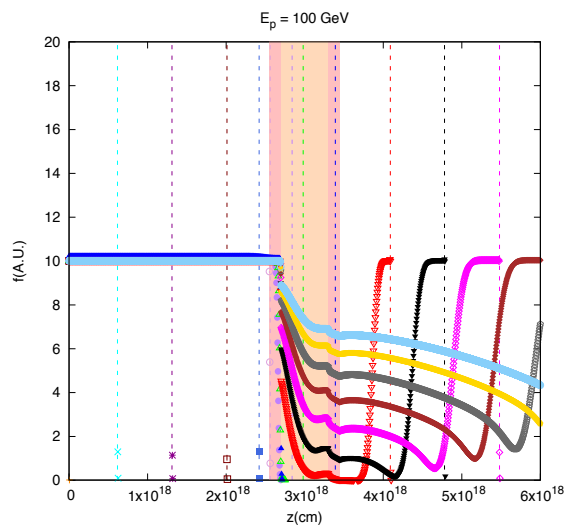

(c)

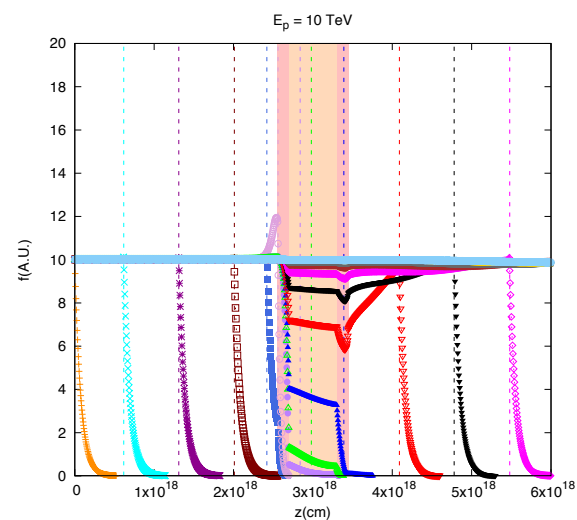

(e)
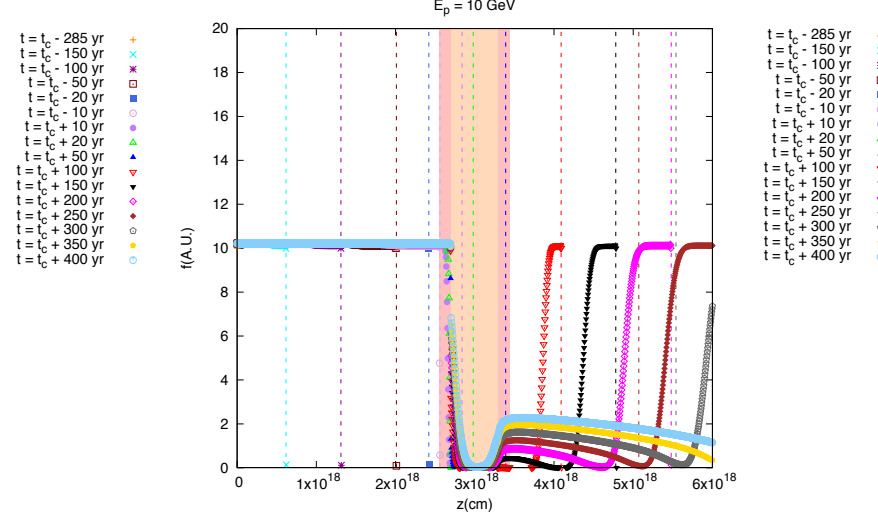

(b)
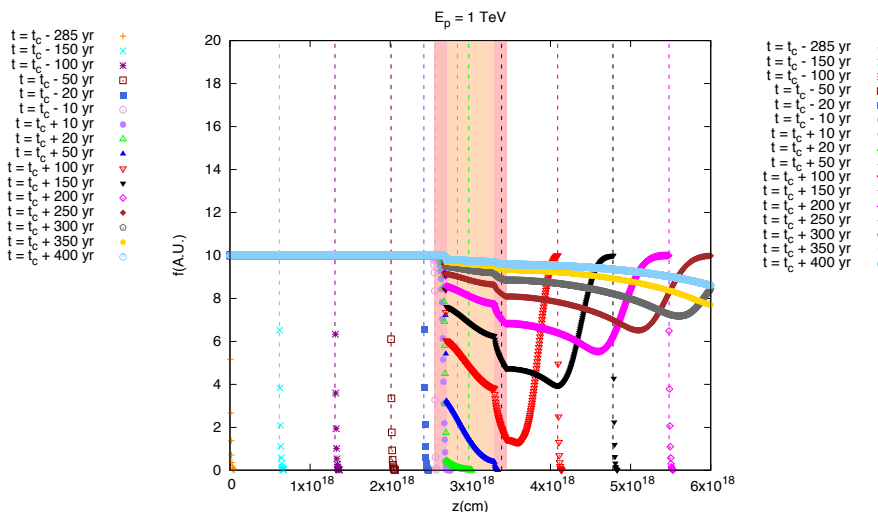

(d)
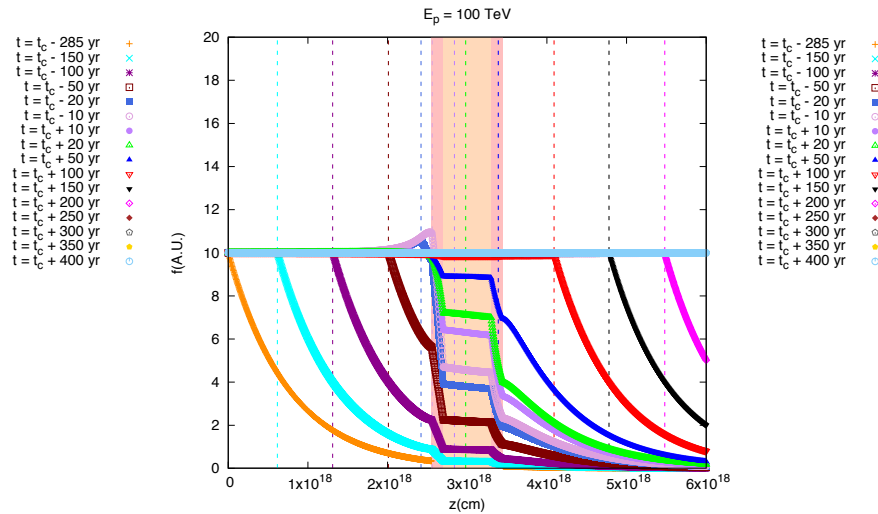

(f)

Figure 1: Distribution function profiles for different CR proton energies, at fixed $r=1.9 \times 10^{16} \mathrm{~cm}$ : the region occupied by the clump, which has a radius $R_{c}=3 \times 10^{17} \mathrm{~cm}$, is shown as a light pink area; the clump layer, characterized by an amplified magnetic field, is the dark pink region surrounding the clump itself. Vertical dashed lines represent the shock position at a given time. (a) $E_{p}=1 \mathrm{GeV}$; (b) $E_{p}=10 \mathrm{GeV}$; (c) $E_{p}=100 \mathrm{GeV}$; (d) $E_{p}=1 \mathrm{TeV}$; (e) $E_{p}=10 \mathrm{TeV}$; (f) $E_{p}=100 \mathrm{TeV}$. 


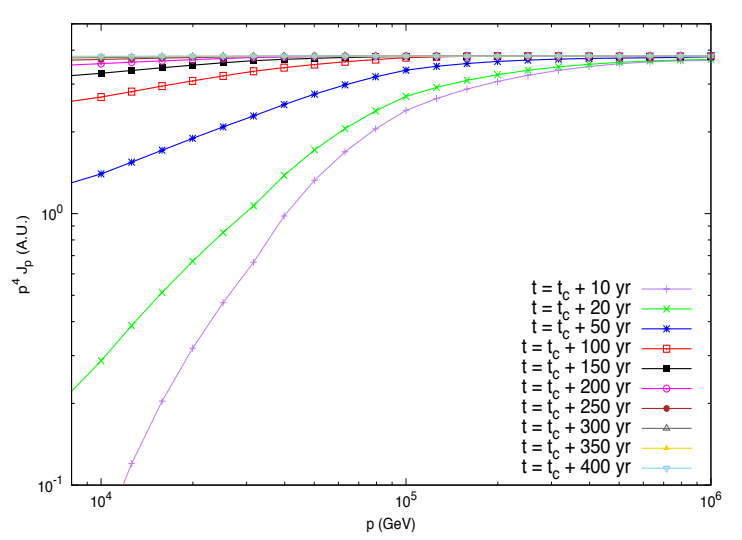

(a)

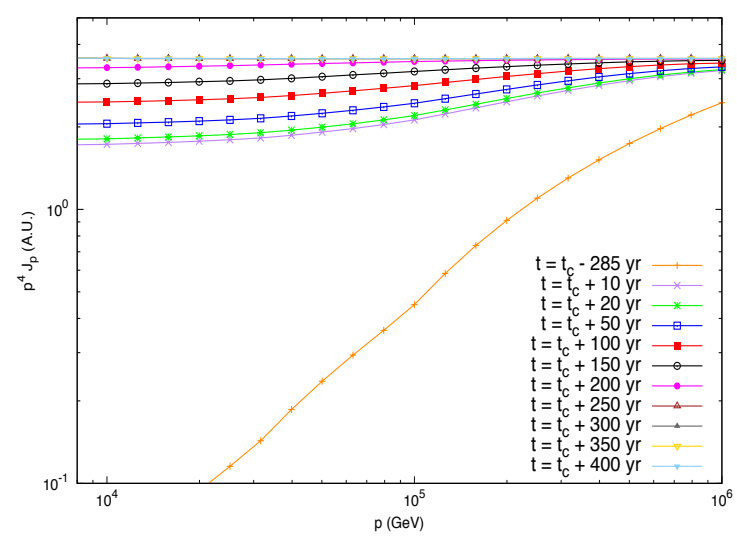

(b)

Figure 2: CR proton spectrum from the region inside the clump (left panel) and from the outer region (right panel), at different times: $t_{c}$ is the time at which the shock enters the clump. The y-axis $p^{4} J(p)$ represents a momentum per unit surface, time and solid angle.

\section{Conclusions}

We studied a scenario where the forward shock of a SNR propagates in a clumpy circumstellar medium. The interaction of the forward shock and the clump results in an amplified magnetic field all around the clump. As a consequence, low-energy accelerated particles do not easily penetrate inside the clump like the high-energy ones and the resulting particle spectrum inside the clump is remarkably harder than the spectrum accelerated at the shock. If the clumps contains most of the target gas, the final $\gamma$-ray spectrum produced in hadronic collisions of accelerated particles can be much harder than the parent spectrum. A possible application of this scenario is constituted by RX J1713.7-3946, since it is believed that the progenitor of this SNR is a massive star embedded in a molecular cloud [7]. The gamma-ray spectra obtained at different times from the shock-clump interaction will be the object of a future work. Furthermore, assuming a spatial distribution of clumps around the remnant will allow to evaluate the clump cumulative contribution to the source emission. Ultimately, a clear understanding of the nature of the detected radiation would come from a multi-messenger perspective, through the identification of astrophysical neutrinos from SNR-MC associations.

\section{References}

[1] M. Ackermann et al. Detection of the Characteristic Pion-Decay Signature in Supernova Remnants, Science 339 (2013) 807 [arXiv:1302.3307]

[2] A. A. Abdo et al., Observations of the Young Supernova Remnant RX J1713.7-3946 with the Fermi Large Area Telescope, ApJ 734 (2011) 28 [arXiv:1103.5727]

[3] V. N. Zirakashvili \& F. A. Aharonian, Nonthermal Radiation of Young Supernova Remnants, APJ 708 (2010) 2 [arXiv:0909.2285]

[4] G. Morlino., E. Amato, P. Blasi, Gamma-ray emission from SNR RX J1713.7-3946 and the origin of galactic cosmic rays MNRAS 392 (2009) 240 [arXiv:0810.0094] 
[5] S. Gabici \& F. A. Aharonian, Gamma-ray emission from young supernova remnants: Hadronic or leptonic?, EPJ Web of Conferences 121 (2016) 04001 [arXiv:1502.00644]

[6] T. Inoue, R. Yamazaki, S. Inutsuka \& Y. Fukui, Toward understanding the cosmic-ray acceleration at young supernova remnants interacting with interstellar clouds: possible applications to $R X$ J1713.7-3946, Astrophys. J. 744 (2012) 71 [arXiv:1106.3080]

[7] S. Gabici \& F. A. Aharonian, Hadronic gamma-rays from RX J1713.7-3946?, MNRAS Lett. 455 (2014) L70-L73 [arXiv:1406.2322]

[8] L. Nava, S. Gabici, A. Marcowith, G. Morlino \& V. S. Ptuskin, Non-linear diffusion of cosmic rays escaping from supernova remnants - I. The effect of neutrals, MNRAS 461 (2016) 4 [arXiv:1606.06902]

[9] R. I. Klein, C. F. McKee \& P. Colella, On the hydrodynamic interaction of shock waves with interstellar clouds. I. Non radiative shocks in small clouds, ApJ 420 (1994) 213

[10] A. R. Bell, The acceleration of cosmic rays in shock fronts, MNRAS 182 (1978) 147

[11] R. D. Blandford \& J. P. Ostriker, Particle acceleration by astrophysical shocks, ApJ Lett. 221 (1978) L29 